\title{
Interfaces da presença familiar no tratamento da criança gravemente enferma
}

\author{
Interfaces of the family presence in the treatment of seriously ill children
}

Interfaces de la presencia familiar en el tratamiento del niño gravemente enfermo

\author{
Gabriele Cieslak $^{1 *}$, Tereza Cristina de Lima Martins², Mari Ângela Berté ${ }^{2}$, Débora Maria Vargas \\ Makuch1.
}

\section{RESUMO}

Objetivo: Descrever as interfaces da presença familiar em contexto de terapia intensiva pediátrica (UTIP) durante o tratamento da criança gravemente enferma. Métodos: Estudo exploratório descritivo, com abordagem qualitativa; coleta de dados se deu por meio de entrevista semiestruturada. Participaram do estudo, 17 familiares de pacientes em UTIP em uma instituição de grande porte exclusivamente pediátrica, no estado do Paraná, entre os meses de Março e Julho de 2020. Para análise das informações, optou-se pela metodologia de Minayo MCS (2007). Resultados: As entrevistas em sua maioria $(70,6 \%)$ foram realizadas em UTIP cardiológica, sendo $64,7 \%$ das participantes mães, com idade média de 18 a 29 anos (53\%) e tempo de internação de 1 até 29 dias (64,7\%). Os discursos entre UTIP cardiológica e UTIP cirúrgica, apresentaram semelhanças emergindo duas unidades de contexto: Presença Familiar em Terapia Intensiva Pediátrica: enfrentamento e adaptação, descreve-se a vivência dos familiares e processos adaptativos a luz de Roy; e a Segurança do Paciente Pediátrico: interface familiar, sendo encontrado discursos de qualidade assistencial. Conclusão: A inclusão efetiva do familiar no processo assistencial permite melhor enfrentamento e adaptação, os familiares coadjuvam com os mecanismos de modulação da resposta hormonal do paciente promovendo melhores desfechos clínicos e melhor resposta terapêutica.

Palavras-chave: Pediatria, Terapia intensiva, Família.

\section{ABSTRACT}

Objective: Describe the family presence interfaces in the context of pediatric intensive therapy (PITU) during the treatment of seriously ill children. Methods: Exploratory descriptive study, with qualitative approach; the data collection was performed through semi-structured interviews. Took part of the study 17 family members of patients in the PITU of a big, exclusively pediatric institution of the state of Paraná between the months of March and July, 2020. For the information analysis, the methodology of Minayo MCS (2007) was chosen. Results: Most of the interviews (70.6\%) were performed in the cardiology PITU, from which $64.7 \%$ of the participants were mothers, with an average age of 18 to 29 years old (53\%) and hospitalization time of 1 to 29 days (64.7\%). The statements of the cardiology and surgical PITUs had similarities between them, including two context units: Family Presence in the Pediatric Intensive Therapy: coping and adaptation, it was described the experience of the family members and the adaptation processes under the light of Roy; and Pediatric Patient Safety: family interface, finding statements of assisting quality. Conclusion: The effective inclusion of family members in the assisting process can help with the coping and adaptation, the family members contribute with the modulation mechanisms of the patient's hormonal response, promoting a better clinical outcome and a better therapeutic response.

Keywords: Pediatrics, Intensive care, Family.

\section{RESUMEN}

Objetivo: Describir las interfaces de la presencia familiar en el contexto de la terapia intensiva pediátrica (UTIP) durante el tratamiento de un niño gravemente enfermo. Métodos: Estudio exploratorio descriptivo, con abordaje cualitativo; recolección realizada a través de entrevista semiestructuradas. Han participado del estudio 17 familiares de pacientes en UTIP de una gran institución exclusivamente pediátrica, del estado de Paraná, entre los meses de marzo y julio de 2020. Para análisis de las informaciones, la metodología de Minayo MCS (2007) ha sido elegida. Resultados: Las entrevistas, en su mayoría (70,6\%), han sido realizadas en UTIP cardiológica, siendo $64,7 \%$ de los participantes madres, con edad media de 18 a 29 años (53\%) y tiempo de internación de 1 a 29 días (64,7\%). Los discursos entre UTIP cardiológica y UTIP quirúrgica presentaron semejanzas, emergiendo dos unidades de contexto: Presencia Familiar en la Terapia Intensiva Pediátrica: enfrentamiento y adaptación, se describe la vivencia de los familiares y procesos de adaptación bajo la luz de Roy; y la Seguridad del Paciente Pediátrico: interfaz familiar, siendo encontrados discursos de calidad asistencial. Conclusión: La inclusión efectiva de los familiares en el proceso asistencial permite un mejor enfrentamiento y adaptación, los familiares auxilian los mecanismos de modulación de la respuesta hormonal del paciente, promoviendo mejores resultados clínicos y mejor respuesta terapéutica.

Palabras clave: Pediatría, Cuidados intensivos, Familia.

\footnotetext{
${ }^{1}$ Faculdades Pequeno Príncipe (FPP), Curitiba - PR. *E-mail: gabycieslak@hotmail.com

2 Hospital Pequeno Príncipe (HPP), Curitiba - PR.
} 


\section{INTRODUÇÃO}

O paciente gravemente enfermo não necessita apenas de cuidados intensivos voltados para sua fisiopatologia, mas também para questões que englobam o universo psicossocial, ambiental e familiar, fatores estes que estão diretamente ligados a recuperação do indivíduo (VILA VSC e ROSSI LA, 2002).

Algumas são as particularidades de uma Unidade de Terapia Intensiva Pediátrica (UTIP), visto que as crianças que dependem de cuidados em terapia intensiva passam por um processo de "separação" de sua família, em um ambiente consideravelmente estranho, o que pode ocasionar estresse emocional devido as mudanças repentinas, mudanças essas relacionadas ao ambiente, considerando o excesso de iluminação, manipulação, ruídos e realização de procedimentos dolorosos e invasivos (GOMES CA e HAHN GV, 2011).

E tratando-se de estímulos dolorosos, o sistema nervoso central é responsável pela sua transmissão até o córtex cerebral. Os recém-nascidos a termo e prematuros sentem a dor com a transmissão mais rápida do que os adultos, nesse público em especifico existe a dificuldade de identificação da dor e de sua intensidade, em razão da criança estar em fase não verbal, a sensação dolorosa ocasionada grava-se como uma memória, ocasionando a diminuição da capacidade de suportar a dor e, ao menor estimulo a criança já sentirá a sensação dolorosa (CORDEIRO RA e COSTA R, 2014).

Apesar de dependentes dos cuidados técnicos, os pacientes em geral também necessitam de pessoas que thes proporcionem conforto e segurança (NASCIMENTO AZ, et al., 2007).

A UTIP pode ser classificada como um ambiente de alto risco onde eventualmente ocorre a comunicação de notícias difíceis, informações essas que não resultam apenas no diagnóstico, mas também dizem respeito as sequelas ou necessidade de cuidados paliativos e em situação de esgotamento de recursos (SOUSA AS, et al., 2018).

O processo de adoecer da criança acarreta em estresse para a família, pois em muitos casos o familiar correlaciona o tratamento intensivo com a aproximação da morte, independente se a patologia é aguda ou crônica. Para a família, o adoecer de um filho traz um universo cheio de sentimentos e incertezas, uma realidade diferente da qual está habituada, e na maioria das vezes esse estágio se apresenta como uma fatalidade para o familiar (HAYAKAWA LY, et al., 2009).

Sabendo das interfaces do tratamento ao paciente pediátrico internado em terapia intensiva, é importante destacar o Estatuto da Criança e do Adolescente (ECA), que descreve o direito da criança permanecer com acompanhante em tempo integral durante a prestação do serviço de saúde, visando a sua melhor recuperação (BRASIL, 2017).

Em vista disso, as instituições de saúde, inclusive as unidades neonatais, UTIP e de cuidados intermediários, deverão ofertar condições para a permanência em tempo integral de um dos pais ou responsável, em casos de internação da criança ou adolescente (BRASIL, 2017).

O conhecimento sobre o enfrentamento da família em contexto de unidade de terapia intensiva é essencial, para que a partir dos dados seja possível engajar métodos atitudinais durante o processo de tratamento da criança gravemente enferma e assim ofertar o cuidado mais humanizado e de melhor qualidade. A partir disto, o objetivo deste estudo é descrever as interfaces da presença familiar em contexto de terapia intensiva pediátrica durante o tratamento da criança gravemente enferma.

\section{MÉTODOS}

Trata-se de uma pesquisa exploratória, descritiva com abordagem qualitativa Participaram do estudo 17 familiares de crianças internadas em UTIP cardiológica e UTIP cirúrgica de uma instituição de grande porte e exclusivamente pediátrica, no estado do Paraná; como critérios de inclusão, foram considerados os familiares maiores de 18 anos, que permaneciam com a criança durante o internamento.

A coleta de dados ocorreu no período de março a julho de 2020, mediante assinatura do Termo de Consentimento Livre e Esclarecido, por meio de entrevista semiestruturada, áudio gravada, com questões inerentes ao levantamento do perfil sociodemográfico dos participantes e com as seguintes perguntas: 
"Como está sendo para você, a vivência dentro da terapia intensiva pediátrica?" "Em sua opinião, a sua presença auxilia ou interfere no tratamento de seu familiar dentro da unidade de terapia intensiva?"

O depoimento de cada participante foi identificado pela letra $E$ (entrevista) e o número correspondente $E 1$, E2 e assim sucessivamente. A análise de informações provenientes da entrevista se sucedeu por meio da técnica de análise de conteúdo, segundo referencial de (MINAYO MCS, 2007).

O desenvolvimento da pesquisa ocorreu atendendo os preceitos da ética, estabelecidos pela resolução CNS 466/2012. Todos os participantes foram informados sobre o objetivo da pesquisa e os métodos adotados para o seu devido desenvolvimento, respeitando acima de tudo a autonomia, liberdade e a dignidade do participante. As abordagens aos participantes ocorreram de forma individual e a entrevista foi realizada em um ambiente privativo para garantir o anonimato e evitando possíveis constrangimentos.

Pesquisa aprovada pelo Comitê de Ética em Pesquisa da Instituição de Ensino Superior sob o parecer de número 3.902.462.

\section{RESULTADOS E DISCUSSÃO}

Quanto à caracterização dos participantes da pesquisa, predominaram Mães correspondendo a 64,7\% seguido de Pais correspondendo a 35,3\%. A faixa etária prevalente foi entre 18 e 29 anos correspondendo a $53 \%$ dos pesquisados. Os demais dados sociodemográficos são descritos na tabela abaixo (Tabela 1).

A maior parte das entrevistas ocorreu na UTIP cardiológica, devido a prolongada permanência dos pacientes. A unidade em questão apresenta diferenças quando comparada com as demais UTIP da instituição, devido ao tratamento intensivo ser voltado para pacientes em estado crítico, onde normalmente a taxa de permanência é de semanas e até meses; em alguns casos mais graves, o paciente é transferido para a unidade logo após ao nascimento para receber o suporte clinico e cirúrgico necessário para sua sobrevida.

Tabela 1 - Variáveis relacionadas ao perfil sociodemográfico dos participantes da pesquisa.

\begin{tabular}{lcc}
\hline Variável & N & $\%$ \\
\hline Parentesco & 11 & \\
\hline Mãe & 6 & $64,7 \%$ \\
Pai & & $35,3 \%$ \\
\hline Idade do Participante & 9 & $53 \%$ \\
18-29 & 8 & $47 \%$ \\
\hline T0-39 & & \\
\hline Tempo de internação & 11 & $64,7 \%$ \\
1 Dia - 29 Dias & 1 & $5,9 \%$ \\
1 Mês - 2 Meses & 4 & $23,5 \%$ \\
Acima de 3 Meses & 1 & $5,9 \%$ \\
\hline Unidade de Terapia Intensiva & & \\
\hline Cardiológica & 12 & $70,6 \%$ \\
Cirúrgica & 5 & $29,4 \%$ \\
\hline Tempo de Permanência & & $23,5 \%$ \\
\hline Até 6 horas & 4 & $70,6 \%$ \\
Até 12 horas & 12 & $5,9 \%$ \\
Tempo integral & 1 & $94,1 \%$ \\
\hline Realiza Revezamento & $5,9 \%$ \\
\hline Sim & 16 & $\mathbf{1 0 0 \%}$ \\
Não & 1 & \\
\hline Total & $\mathbf{1 7}$ & \\
\hline
\end{tabular}

Fonte: Cieslak G, et al., 2020. 
Percorrendo as etapas preconizadas por Minayo MCS (2007), após a exploração dos discursos dos participantes emergiram duas unidades de contexto: Presença Familiar em Terapia Intensiva Pediátrica: enfrentamento e adaptação; Segurança do Paciente Pediátrico: interface familiar.

\section{Presença familiar em terapia intensiva pediátrica: enfrentamento e adaptação}

Após a confirmação da patologia e a necessidade de atendimento da criança em UTIP, alguns papéis familiares e funções sociais podem sofrer reorganização, e invariavelmente apresenta-se a necessidade de interrupção de algumas funções causando assim, instabilidade no contexto familiar. A família vivencia uma série de sentimentos frente ao desconhecido, como medo e insegurança, diante das mudanças e consequências impostas pelo tratamento (ANJOS C, et al., 2019).

Entender toda a transição no processo de saúde-doença em que a criança se encontra é de demasiada importância, para que o profissional enfermeiro articule os modelos clássicos de cuidados em enfermagem, e assim promova uma melhor vivência deste processo.

Sabendo que o adoecer da criança traz alterações não apenas no contexto familiar, mas também na forma com que o indivíduo interage em seu meio psicossocial, o modelo teórico de Callista Roy transcreve sobre o intento da Enfermagem proporcionar formas para adaptação deste indivíduo (COELHO SMS e MENDES IMD, 2011).

Nos discursos apresentados pelos familiares, emanam o medo do desconhecido sobre a internação da criança em UTIP.

"É muito intenso devido ao estado da minha filha, ela está em estado grave, intenso no sentido psicológico, físico, (...)" (E1).

"Para ser bem sincero não está sendo muito fácil devido a situação dela e tudo, eu espero que logo ela vá melhorando né " (E2).

"A gente entra em uma UTI e não tem certeza de como é um tratamento intensivo" (E10).

Os familiares vivenciam desconfortos de origem física, psíquica e social, resultantes das interações ocorridas no ambiente em que atualmente se encontram, além do processo de enfrentamento da doença e a adaptação ao cuidado de uma criança internada em UTIP (FREITAS KS, et al., 2012).

Contudo, os sentimentos resultantes da vivência desta situação única, faz com que o familiar conceba a inevitabilidade de estar presente e proporcionar conforto, para que assim, consiga contribuir com o tratamento da criança (NASCIMENTO AZ, et al., 2007).

“(...) às vezes ela está agitada, com dor e a saturação começa a cair e o batimento cardíaco sobe, nesse momento eu falo com ela, toco nela e ajuda a melhorar esses sinais, ela se acalma e a saturação melhora" (E7).

"No meu ponto de vista eu acho que faz bem eu estar ali, mesmo ela estando com o tubo, com dreno, cheia de acesso, quando eu chego e converso com ela, ela ri" (E4).

"A minha presença auxilia no tratamento do meu filho, na questão de acalmar ele, de ficar mais próximo dele, ele sente a presença dos pais ali não se sente sozinho, $e$ eu acho que isso ajuda bastante na questão do tratamento, quando ele está agitado, ao toque ou ao ouvir minha voz ele já fica mais calmo" (E3).

No passado considerava-se que recém-nascido não possuía captação do estimulo doloroso devido imaturidade do sistema nervoso, recentes estudos desvelam que a diminuição de fibras mielinizadas está diretamente relacionada a transmissão mais rápida do estimulo álgico (PEREIRA LSS, et al., 2019). A percepção mais rápida do estimulo doloroso, assim como demais sinais de desconforto, como, fome, ansiedade ou alterações em sua patologia de base, o paciente pode apresentar alterações secundárias ao 
estímulo, manifestadas na frequência cardíaca, frequência respiratória, pressão arterial, saturação, vasoconstrição periférica, sudorese, dilatação pupilar além do aumento na liberação de catecolaminas e hormônios adrenocorticosteróides. Tais sinais e sintomas estão ligados ao mecanismo compensatório do sistema nervoso central autônomo, o qual é ativado como resposta a diversos estímulos (SILVA YP, et al., 2007).

O córtex insular posterior é um dos responsáveis no processo de percepção de sensações álgicas. Uma vez que a criança está diante de um estimulo doloroso, há elevação de catecolaminas e adrenocorticosteróides, o que levará a alterações nos sinais vitais (elevação pressórica, aumento na frequência cardíaca, dentre outros já citados). Ao ser tocada ou ao ouvir a voz dos pais, estimulo consideravelmente prazeroso e agradável, ocorre a ativação do córtex insular anterior esquerdo ocasionando a redução da frequência cardíaca e pressão arterial. Diante disso, a presença do familiar durante o internamento deve ser considerada (JABBI M, et al., 2007).

Do ponto de vista fisiológico, a presença do familiar com a criança enferma é correlacionada com a modulação da resposta hormonal do paciente, a qual auxilia na diminuição do estado de alerta. (BRASIL, 2007). Aplicar medidas para controle e alivio da sensação dolorosa está inerentemente ligada a qualidade assistencial, além de promover medidas adaptativas do indivíduo frente a vivência hospitalar (PEREIRA LSS, et al., 2019).

"Eu sempre tento estimular a mente dele pois até onde eu sei ele está nessa fase de formação e eu tento estimular funções com desenhos e cores(...)" (E6).

"Eu passo segurança para ela, eu acho que ela sente um pouco da energia." (E8).

"O sentimento, primeiro é uma empatia pelo profissional da saúde para ver como ele está tratando do seu bem mais precioso que está ali em terapia intensiva, e ao mesmo tempo o como você tenta ajudar, ajudar com o carinho e com um pouco de atenção. " (E5).

Nos discursos é possível depreender a importância que o familiar atribui à sua permanência na UTIP acompanhando a criança gravemente enferma sendo evidente o processo adaptativo que o familiar vivenciou; para tal, a atuação da equipe de enfermagem é essencial, na promoção de uma assistência segura e na efetivação de protocolos de boas práticas, além do suporte emocional ofertado, pois dentre as profissões da saúde, o profissional de enfermagem é aquele que mais tempo permanece com o paciente, o que inevitavelmente permite um maior vínculo nas relações de cuidado e o conforto ao familiar acompanhante. Inicialmente os discursos apresentaram sentimentos de medo sobre o desconhecido, e posteriormente os familiares iniciaram com falas onde é possível evidenciar o processo de adaptação ao ambiente onde atualmente se encontram-se, percebendo assim, o sentido e a beneficência de sua presença com a criança hospitalizada em UTIP.

A prática de enfermagem desenvolve promoção em saúde, pela qual os cuidados são focados nos processos da vida humana; auxiliando assim, o processo da adaptação do indivíduo frente ao momento vivido. Pode-se descrever tal processo utilizando metaparadigmas onde englobam-se os seguintes conceitos: a pessoa; o ambiente e a saúde (COELHO SMS e MENDES IMDM, 2011).

À luz de Roy, a pessoa é um centro adaptativo constituída por elementos, sendo o Input representado pelos estímulos internos e externos; output que é formado por respostas diante do enfrentamento; e Feedback. Os argumentos demonstram estímulos aos quais os familiares estão expostos, estes sendo contextuais, focais e ambientais, todos relacionados ao estado clínico da criança em tratamento na UTIP. (GUEDES MVC e ARAÚJO TL, 2005).

O processo adaptativo do familiar ocorrerá de forma positiva ou negativa, consequência dos estímulos internos e externos descritos anteriormente, porém cabe ao profissional enfermeiro a aplicação de medidas estratégicas e de boas práticas no desenvolvimento assistencial, o cuidado centrado na família, desta forma, favorecendo e contribuindo para a qualidade do serviço e elevando o nível de satisfação e segurança do paciente (WEGNER W, et al., 2017). 
Estudos evidenciam que as relações de cuidado estabelecidas pela equipe de enfermagem, favorecem o processo adaptativo dos envolvidos, além de proporcionar conforto e segurança para o familiar. $O$ conhecimento técnico científico articulado as habilidades de comunicação e de empatia da equipe, possibilitam que o familiar vivencie e provavelmente sublime as dores e sofrimentos deste momento singular (ALVES VS, et al., 2011).

As percepções dos familiares quanto as relações de cuidado no contexto de terapia intensiva pediátrica, também são descritas na unidade de contexto a seguir.

\section{Segurança do paciente Pediátrico: Interface familiar}

A interação do acompanhante com a equipe multidisciplinar, além de oferecer mecanismos de enfrentamento para o familiar, constitui uma forma de segurança para o paciente, proporcionando benefícios no atendimento. A temática frente a segurança do paciente é algo que vem sendo discutido há algum tempo, é de grande importância, pois aplica métodos que proporcionam a redução de possíveis riscos ou danos ocasionados durante a assistência em saúde (SILVA T, et al., 2012).

É necessário desenvolver empatia pelo familiar, e cabe à equipe envolvê-lo no processo de atenção a criança, comunicando-o sempre que for realizar algum procedimento além de deixar o familiar ciente do quadro clínico da criança. Para tal ação, a equipe de enfermagem deve-se respaldar de conhecimento técnico cientifico, para que assim consiga ofertar uma assistência planejada e estruturada, realizando orientações e captando necessidades que o familiar venha apresentar (MARQUES RC, et al., 2009).

"A gente participa também na conferência dos medicamentos, vendo nome... então isso para mim faz diferença, e qualquer sinal de alerta a gente já comunica os técnicos que entravam em contato com os médicos" (E1).

"Estou sempre acompanhando os médicos e as enfermeiras, aí todo dia eles falam o que eles fazem, eu me sinto importante ali" (E9).

"Vejo os remédios que ele vai tomar, a enfermagem sempre pede para eu ver o remédio, o nome completo dele, o horário e ver também a folha que o médico pediu aquele remédio, acho que é importante isso" (E 11).

"A gente ali como pai acaba sendo guardião do nosso filho" (E5).

É possível inferir pelos relatos capturados que os familiares sentem segurança frente as tecnologias utilizadas na UTIP, entretanto desvelam que sua presença é indispensável, para que a criança consiga se sentir segurança dentro de um ambiente diferente de seu habitual. (NASCIMENTO AZ, et al., 2007). A segurança como é relatada, evidencia a aplicação satisfatória de políticas de qualidade assistencial e segurança ao paciente, o que se dá por várias vertentes, uma delas é a comunicação efetiva, onde o profissional envolve o familiar no processo de assistência à criança, sempre comunicando-o sobre os procedimentos e medicamentos a serem realizados; o familiar também representa um papel importante dentro da assistência ao paciente, onde ele mostra-se como barreira para prevenção de possíveis erros.

A efetividade da comunicação dentro da UTIP é um fator determinante para monitorizar a qualidade e segurança do atendimento prestado ao paciente. A comunicação deve ser realizada de forma efetiva e clara por todos os profissionais envolvidos no cuidado ao paciente, devendo esclarecer dúvidas e orientando sobre os procedimentos a serem realizados, com vistas à minimização dos riscos além de inserir a família no processo de assistência à criança hospitalizada (SILVA T, et al., 2012).

A desconsolação dos familiares, desencadeada pelo internamento de uma criança na UTIP pode ser minimizada quando a família se torna sujeito da atenção à saúde, como preconizam as políticas e os discursos de humanização do Ministério da Saúde (BRASIL, 2007). A aplicação sistêmica e continua das políticas de qualidade e segurança, implementando recursos de identificação e analise dentro dos serviços de saúde, possibilita o controle de possíveis riscos e eventos que venham a afetar a saúde e a integridade do paciente, cabe a toda equipe a implementação de protocolos de segurança do paciente, desta forma permitindo a redução de eventos adversos relacionados a assistencia em saúde, o que proporciona uma maior seguridade ao paciente e a seu familiar (BRASIL, 2015). 
Comparado com estudos recentes, é perceptível que a atuação dos profissionais responsáveis pela assistência ao paciente é desenvolvida de forma humanizada e com aplicação efetiva das medidas de segurança para evitar possíveis erros ou danos em saúde. Silveira GFC, et al. (2019) relata a sua experiência dentro de UTIP, onde, familiares ficam longe de seus filhos devido ao tratamento intensivo, deixando-os aos cuidados da equipe multiprofissional. O distanciamento do familiar durante a internação, além de ocasionar desconfortos emocionais, está relacionado ao estado clínico da criança, pois como citado anteriormente, a presença do familiar auxilia na liberação hormonal e consequentemente contribui para melhor aceitação da terapia, além de ser mais uma barreira de segurança.

\section{CONCLUSÃO}

Os resultados do estudo apresentaram a percepção e as interfaces do familiar em contexto de terapia intensiva pediátrica durante o tratamento da criança gravemente enferma, desvelando inicialmente o medo e insegurança frente ao desconhecido e também ao elevado temor da perda (óbito), e que a inclusão efetiva do familiar no processo assistencial permite um melhor enfrentamento e adaptação além de promover a segurança do paciente como uma medida adicional de prevenção para possíveis erros. A equipe de enfermagem possui um grandioso papel, onde além de realizar os cuidados, oferta medidas para que 0 familiar sinta-se importante durante todo o processo. Destacou-se também, que os familiares coadjuvam com os mecanismos de modulação da resposta hormonal. Assim, a presença do acompanhante promove melhores desfechos clínicos e melhor resposta terapêutica.

\section{REFERÊNCIAS}

1. ALVES VS, et al. Conhecimento de profissionais da enfermagem sobre fatores que agravam e aliviam a dor oncológica. Revista Brasileira de Cancerologia, 2011; 57(2):199-206

2. ANJOS C, et al. A permanência da família no centro de terapia intensiva pediátrica oncológica: percepção da enfermagem. Rev Min Enferm., 2019; 23: e-1180.

3. BRASIL. Ministério da Saúde. Lei Federal № 8,069 de 13 de julho de 1990, Estatuto da Criança e do Adolescente. ECA Versão atualizada. Brasília 2017.

4. BRASIL. Ministério da Saúde. Secretaria de Atenção à Saúde. Núcleo Técnico da Política Nacional de Humanização. Humaniza SUS: visita aberta e direito a acompanhante / Ministério da Saúde, Secretaria de Atenção à Saúde, Núcleo Técnico da Política Nacional de Humanização. 2. ed. Brasília: Ministério da Saúde, 2007.

5. BRASIL. Plano integrado para a gestão sanitária da segurança do paciente em serviço de saúde. Política Nacional de Segurança do Paciente. Brasília: Ministério da Saúde, 2015.

6. COELHO SMS, MENDES IMD. Da pesquisa à prática de enfermagem aplicando o modelo de adaptação de Roy. Esc Anna Nery (impr.), 2011; 15 (4): 845-850.

7. CORDEIRO RA, COSTA R. Métodos não farmacológicos para alívio do desconforto e da dor no recém-nascido: uma construção coletiva da enfermagem. Texto Contexto Enferm, Florianópolis, 2014; 23(1): 185-92.

8. FREITAS KS, et al. Desconfortos vividos no cotidiano de familiares de pessoas internada na UTI. Esc Anna Nery (impr.), $2012 ; 16$ (4): $704-711$.

9. GOMES CA, HAHN GV. Manipulação do recém-nascido internado em UTI: alerta à enfermagem. Revista destaques acadêmicos, 2011; 3: 3.

10. GUEDES MVC, ARAÚJO TL. Crise hipertensiva: estudo de caso com utilização da classificação das intervenções de enfermagem para alcançar respostas adaptativas baseadas no Modelo Teórico de Roy. Acta Paul Enferm., 2005; 18(3): 241-6.

11. HAYAKAWA LY, et al. Alterações familiares decorrentes da internação de um filho em uma unidade de terapia intensiva pediátrica. Rev. Gaúcha Enfermagem, 2009; 30(2): 175-82.

12. JABBI M, et al. Empathy for positive and negative emotions in the gustatory cortex. Neuolmage Elsevier. 2007; 34: $1744-1753$.

13. MARQUES RC, et al. Comunicação entre profissional de saúde e familiares de paciente em terapia intensiva. Rev. enferm. UERJ, Rio de Janeiro, 2009; 17(1): 91-5.

14. MINAYO MCS. O desafio do conhecimento: pesquisa qualitativa em saúde. 10.ed., São Paulo: Hucitec, 2007.

15. NASCIMENTO AZ, et al. Limites e possibilidades da permanência de familiares em unidade de terapia intensiva. Cogitare Enfermagem, 2007; 12(4): 446-51.

16. PEREIRA LSS, et al. Conhecimento dos profissionais de enfermagem na avaliação da dor neonatal em uma unidade de terapia intensiva. Revista Eletrônica Acervo Saúde, 2019; 11(14): e1122.

17. SERRANO SC, et al. Peculiarities of neuropathic pain in children. Rev Dor, 2016; 17(Suppl 1): S110-2.

18. SILVA T, et al. Segurança da criança hospitalizada na UTI: compreendendo os eventos adversos sob a ótica do acompanhante. Revista Eletrônica De Enfermagem, 2012; 14(2): 337-44.

19. SILVA YP, et al. Avaliação da Dor em Neonatologia. Revista Brasileira de Anestesiologia 2007; 565(57): 5.

20. SILVEIRA GFC, et al. Unidade de terapia intensiva pediátrica: sentimentos maternos frente à hospitalização. Revista Eletrônica Acervo Saúde, 2019; 31: e1139.

21. SOUSA AS, et al. Experiências maternas diante da transferência do filho para a unidade de terapia intensiva. Rev baiana enfermagem, 2018; 32: e25160.

22. VILA VSC, ROSSI LA. O significado cultural do cuidado humanizado em unidade de terapia intensiva: muito falado e pouco vivido. Rev Latinoam Enfermagem, 2002; 10(2): 137-44.

23. WEGNER W, et al. Segurança do paciente no cuidado à criança hospitalizada: Evidências para a enfermagem pediátrica. Rev. Gaúcha Enferm, 2017; 38: 1. 